\title{
Interactive Virtual Reality Touring System: A Case Study of Shulin Ji'an Temple in Taiwan
}

\author{
Jung-Hua Lo $\mathbb{D},{ }^{1}$ Shih-Da Wu $\mathbb{D},{ }^{2}$ and Min-Jie You $\mathbb{D}^{1}$ \\ ${ }^{1}$ Department of Applied Informatics, Fo-Guang University, Yilan 26247, Taiwan \\ ${ }^{2}$ Management College, National Defense University, Taoyuan City 11258, Taiwan \\ Correspondence should be addressed to Jung-Hua Lo; jhlo@mail.fgu.edu.tw
}

Received 31 October 2020; Revised 7 August 2021; Accepted 17 August 2021; Published 25 August 2021

Academic Editor: Hsu-Yang Kung

Copyright (c) 2021 Jung-Hua Lo et al. This is an open access article distributed under the Creative Commons Attribution License, which permits unrestricted use, distribution, and reproduction in any medium, provided the original work is properly cited.

Most current tour guiding methods for Taiwanese temples employ graphic webpage frameworks combined with captioned pictures for introduction. This type of tour guiding lacks interactive presence. In addition, the audience may not be able to focus on browsing webpages or learn essential information from the introduction. This study adopted the Delphi method to evaluate the current developed system. This system was aimed at designing VR-based interaction that differs from conventional tour guiding methods to aid users in viewing the display space from their viewpoints. Users cannot only control camera view angles but also select the paths and guiding information as if they were walking in the temple. The analysis results revealed that, in general, the users perceived VR tour guiding as convenient and easy to use. The display and content of the tour guiding system presented clear information to the users, aiding them in gaining further understanding of the introduced item. Finally, the study results can serve as a reference for design research on VR applications in tour guiding.

\section{Introduction}

1.1. Research Background and Motivation. Religious activities in Taiwan are the most authentic cultural expression of the lives of the common people, combining the beliefs of Buddhist and Taoist deities and the rituals of heaven and ancestor worship, which are rich and diverse and reveal the deep cultural assets of Taiwan. A variety of temple fairs, temple parade techniques, and temple architecture and craftsmanship, based on religious beliefs, demonstrate the abundance of local culture. In recent years, many of the local activities promoted by the government have been aimed at attracting tourists by integrating the local tourism industry with the culture of religious beliefs as a regional feature. As a result, religious and cultural tourism is not only fostered in the region, but has also become a popular tourist activity. In the case of the Jian Temple in New Taipei City, this study, by examining the attractiveness of religious tourism in the process of promoting cultural tourism at regional temples, has developed a tour guiding system at the temple, making use of the temple's existing resources and textual and pictorial descriptions to convey the unique local culture of Taiwan. Through daily contact, the temple naturally helps the worshippers and visitors to understand the history of the area and also imperceptibly enhances their cultural literacy so that the shrine is no longer just a center of faith, but also functions as a local exhibition hall, with the aim of boosting local cultural tourism. This kind of experience will help visitors to get a better sense of the culture of the Taiwanese people, and it is only through understanding the culture of Taiwan that they will be able to bond and identify with the land. Temples embody the living habits, customs, outlook on life, and values of the locals and exhibit a wide range of cultures. In addition, the loss of temple culture in Taiwan has prompted this study to delve into the cultural tourism of temples and to develop a tour guiding system for temples.

Tourist attractions usually present information through guided tours, documents, brochures, information plaques, and audio tours. These methods can fail to present visitors with accurate and precise information because of inadequate human resources or outdated text and audio materials. Such situations can leave visitors with poor impressions of the interactions or 
services offered by attractions. With the rapid advancement of the Internet technology, the daily life of most people is becoming strongly entangled with the Internet and relevant technology applications. That is, the Internet has changed people's lifestyle and consumption habits. Technological advancement and the widespread use of personal intelligent mobile devices, such as smartphones and tablets, have enabled visitors to locate nearby tourist attractions directly using the camera and location-based services (LBS) of their mobile devices. The popularity of smartphones that offer a wide variety of application software (colloquially referred to as APP) in addition to basic telephone, Internet, and gaming functions has given rise to a new generation of APP that are armed with interactive features. Such interactive features, which may involve text, images, audio, and video, create an innovative user experience. Some have even incorporated another emerging technology that is growing in popularity: virtual reality (VR). VR's greatest selling point is that it enables users to experience a world in which the real and the virtual are seamlessly integrated $[1,2]$; additionally, it allows users to interact with this world through certain ancillary devices (e.g., the display device) and experience the integration between the virtual and real worlds while controlling virtual items in the real-world environment. However, most mobile applications that offer tour services do not have a correct understanding of the places they are visiting due to lack of time, failure to read maps, or lack of tour guides. Or, the information of the tourist attraction is not up to date, which leads to incorrect information at the first place and in turn misunderstanding about the places they are visiting. Also, the mobile-guided tour APP available in the market are designed mostly with the general public in mind. There is still plenty of room for improvement. Based on the aforementioned reasons, we develop VR mobile guide system operated on smart phones through the corresponding scheme of integration with GPS function can be easily used without any geoinformation provided by Internet. This mobile guide system can be known as a personal-oriented LBS integrated with Google e-map, for users to use smart phones with GPS function as a guide tool, guiding users based on the routes chosen by users. The functions include query for nearby scenic areas, introduction to the content of scenic areas through different dimensions, presentation of photos on the history of scenic areas, spatial guide on e-map, and introduction of augmented reality direction of scenic areas. On the contrary, as digital technology advances and the smart mobile devices from launch to availability, mobile guide services have progressed to the interaction with reality information guide following the past static guide scheme. The study integrates jQuery Mobile, VR techniques, LBS, and other technologies to develop an VR mobile guide system so that users can find the information on the target destination in reality via the screen of smart phones to carry out the integration of reality guide and route guide functions, thereby providing reference and application for the establishment of the future mobile guide system [3-5].

1.2. Research Questions. This study focused on Ji'an Temple of Shulin District, New Taipei City, Taiwan. An interactive tour guiding of the temple was thus developed through VR technologies. The Delphi method, which is an expert group decision-making technique, was employed on the basis of multiple rounds of questionnaire responses. Through cyclic feedback collection, various experts' comments were collected. Indicators that experts consistently agreed are critical in VR applications in interactive tour guiding were selected as the goal orientation for system development. Thus, this study was conducted to respond to the following three questions and also shown in Figure 1:

(Q1) What are the major dimensions and indicators established for the target touring mobile information system from the expert opinions?

(Q2) How to develop the target touring mobile information system that meets the indicators recommended by the expert opinions?

(Q3) The users' responses are important. What is the result of the Questionnaire for User Interaction Satisfaction (QUIS)?

The rest of this paper is organized as follows. In Section 2, the literature review of temple cultural tourism and VR technology is discussed. The expert assessment of the system was evaluated in Section 3. VR mobile touring system framework and development is proposed in Section 4 . The questionnaire for user interaction satisfaction is discussed in Section 5. Finally, the conclusions and future studies are described in Sections 6 and 7, respectively.

\section{Literature Review}

In this section, we first introduce the Ji'an Temple and discuss the temple cultural tourism in Taiwan. Then, the VR applications installed on mobile devices for temple guidance are reviewed.

2.1. Temple Cultural Tourism and Ji'an Temple. Under the impact of urbanization, traditional folk culture and arts are facing a great dilemma in terms of preservation, and Taiwan's local culture is gradually in danger of disappearing, and temples are almost becoming the last reserves of these folk cultures and arts. The protection of temple culture is a way to preserve Taiwan's intangible cultural heritage so that these valuable folk arts can be passed on through folk beliefs and local temples can sustain operations through the boost of tourism. The continued conservation of folk art and culture creates a sense of identity and continuity and promotes the development of cultural diversity and pluralism. Intangible cultural heritages are a treasure shared by all people in Taiwan. They are the gems of wisdom from the ancestors' lives, an important asset in shaping Taiwan's unique style in a culturally homogenized world and an essential medium for bringing Taiwan's cultural content and characteristics to international attention $[6,7]$. This study, by using the example of the Ji'an Temple in Shulin, aims to look into its background to learn about the past with literature review, and by collecting the characteristics of religious tourism and organizing and analyzing the factors driving tourists to engage in religious and cultural sightseeing tour, 


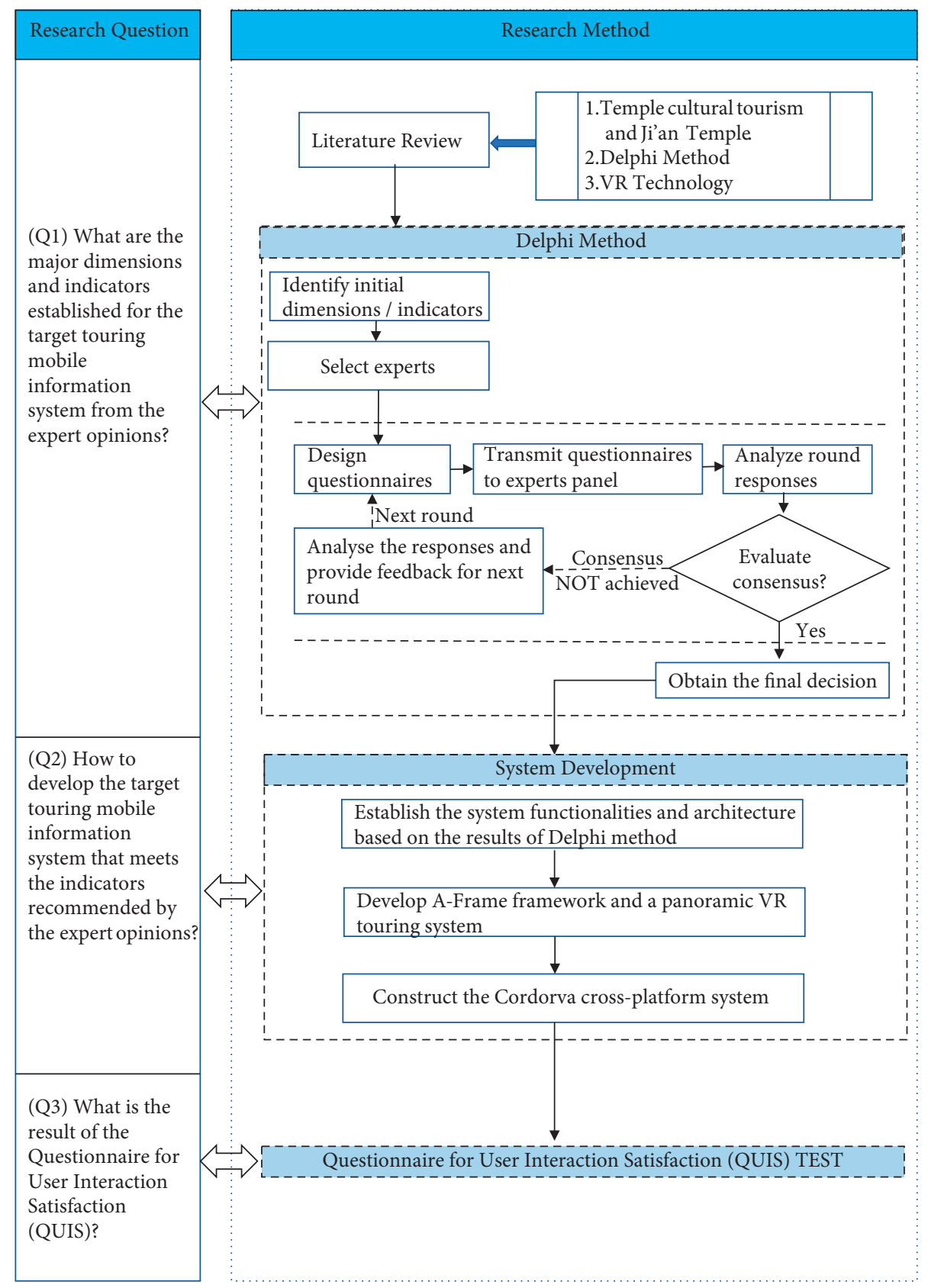

Figure 1: Research question statement.

it can, based on the analyzed data, identify the specific direction for development and serve as a basis for projecting the development of the functions of the tour guidance system. We have gathered information about the people, events, objects, and environment concerning the Jian Temple through historical and documentary materials, which have served as a reference for the planning and design of the guiding system, and then collected books, documents, cultural and historical materials, and data on websites related to the shrine, in order to gain a preliminary understanding of the historical background and local culture, as well as to find out the historical and cultural references of the shrine and to use these materials as a basis for research. Established over 230 years ago, the Jian Temple in Shulin,
New Taipei City, is the most representative shrine in the Shulin area. The Jian Temple is dedicated to Bao-SBheng Emperor [8-10], a deity in charge of medicine, who is well versed in astronomy and geography and is a skilled healer, specializing in the treatment of maladies and saving countless lives. It is said to be so effective that, in later times, he was revered as the "God of Medicine," a deity to whom people pray for good health. One of the most special features of all temples worshipping Bao-Sheng Emperor is that there are divination prescription slips available in the temple. Therefore, for patients or their families, they can come to the temple to pray for the protection of the gods and also to seek prescriptions for medicine and then get medicines from the Chinese medicine shop and give to the patients. In general, 
this type of prescription slips is no different from drawing a bamboo divination stick to ask for good fortune. However, before drawing the slip, apart from praying, one must also give a statement of one's condition to the deity and then draws a bamboo stick from the stick box and throws divination blocks to determine whether it is suitable for a prescription. Since the belief in healing is more or less helpful to the illness, the belief in Bao-Sheng Emperor has persisted for hundreds of years, making the temple building, sacrificial items, and festivals distinct from other shrines and becoming a unique temple in the region. This study aims at exploring the cultural tourism resources of temples from the perspective of religious tourism, and through the example of the Jian Temple, a guided tour system of the temple has been built to convey the unique local culture of Taiwan and to probe into its temple architecture, religious impressions, etc. By virtue of the impression and attractiveness of the destination, this study analyses the advantages of the development of religious and cultural tourism and uses a virtual model to integrate the tour system into the temple so that, through the explanation of specific displays and ritual activities in the temple, the visitors and worshippers can be impressed and moved, with the purpose of transmitting cultural messages and interpreting cultural language. In addition to preserving the cultural assets and linking them to the past, it also serves to pass on the wisdom of the ancestors and to explain the history and culture of the community so that the worshippers and visitors do not only hold incense sticks and worship on a religious sightseeing tour, but through the guided tour system, understand the reasons for worshipping, the origins of the faith, the history, and the importance of passing it on.

\subsection{VR Technology. Gartner's Top 10 Strategic Technology} Trends for 2020 [11] mentions VR and AR as one of the top 10 emerging technologies to watch in the coming years. VR is a simulated space and environment that can be made to feel like a real environment. VR can fully simulate human perception, allowing visitors to have an almost realistic experience [12-14]. In other words, a computer-generated virtual world in three dimensions enables the device to perform complex computations immediately when the user moves around, sending back an accurate $3 \mathrm{D}$ image of the world to create a sense of presence so that the user can see things in three dimensions in a timely and unrestricted way, as if they were there. These fictional worlds feature, to varying degrees, immersion, interaction, and imagination [15], created by a simulation system that integrates computer graphics technology, computer simulation technology, artificial intelligence, sensing technology, display technology, and parallel network processing. VR can be divided into the following three categories according to the way it is created: geometry-based VR, image-based VR, and hybrid VR, which are introduced below [16-23]:
(1) Geometry-based VR: geometry-based VR scenes and objects are computed by computers, and the objects are created using computational geometric operations and mapping techniques to produce the socalled VR. Generally, 3D modelling tools (e.g., 3ds Max, Maya, and AutoCAD) are utilized to create the scenes, and then, different software is applied to build the required virtual environment depending on the needs on-site. Using various VR editing software (e.g., Unity), objects are imported and set with different attributes, and trigger events are added to the model objects as appropriate, as a way to communicate messages and show event-triggered interaction to achieve engagement with virtual scenes. This kind of VR production is time consuming and requires a lot of manpower to build the virtual environments, but the final result is very interactive and can be viewed from any angle and path.

(2) Image-based VR: real images are used based on computer technology to create a virtual world that is comparable to our real environment. Since geometry-based VR is relatively expensive and takes longer to create, image-based VR, built on panoramic image technology, was created as a result. Image-based VR uses a camera, a tripod, and image-based VR editing software to present the viewer's surroundings in a cylindrical or spherical field of vision as a still image in VR. Today's digital cameras are evolving rapidly, making image-based VR production even easier. Developers can create the most realistic virtual scenes in a fraction of the time.

(3) Hybrid VR: hybrid VR, by adding virtual objects to real images, is a technology that lies somewhere between geometry-based VR and image-based VR, with some of the advantages and disadvantages of the former two, but strikes a balance between them. The first step is to create a geometric virtual scene to simulate a real setting, but often due to the complexity of modelling the interior, a video virtual tour is adopted to complement the geometry and make the whole scene complete.

Geometry-based method can provide users with high interactivity and high immersion, but using this method to develop highly realistic artificial environment is time-consuming. In contrast, the image-based method can produce highly realistic artificial environment in a shorter time, making it a possible alternative to the geometry-based method. The virtual effect of the image-based method is to use the images of photos to be stitched together to develop a realistic panoramic virtual environment. Furthermore, it can use zoom in/out edges in artificial environments and perform hot-spot jumps and 360-degree panning between several artificial environments to achieve navigation. 
Compared with the geometry-based method, the navigation function developed using the image-based method is limited. Especially, when the scale of the scene is large or the surface details of the objects in the scene are more complicated, the time consumed for modelling and rendering will increase sharply, and the requirements for hardware will also be relatively high. Moreover, VR provides virtual navigation of the environment and breaks the boundary between the virtual and the real, allowing users to combine highly interactive manipulation with the real environment, creating sensory stimulation and retaining an interactive, personalized experience. VR uses the technology of computers and displays to generate a 3D virtual world. Most of the current VR-related devices are designed with displays that completely envelop the space around the eyes, creating a seemingly realistic 3D virtual world in the user's field of vision. In addition, users can also combine sensors to control objects in the virtual world. The computer learns the user's body movements through the sensors and generates corresponding images to achieve an interactive situation with the user. Panoramic image navigation is one of the VR applications, and these technologies are becoming increasingly sophisticated, and the cost and technical barriers are not so high that users can quickly immerse themselves in the environment. Many of the country's tourist attractions now also adopt the panoramic image navigation system for guidance. For example, the Bureau of Cultural Heritage has developed a tangible cultural assets tour and management system that uses geo-information technology to incorporate spatial databases of tangible cultural assets in Taiwan, allowing users to collect information on scenic spots and make online enquiries, while integrating mobile devices to provide 360-degree panoramic tours so that the general public can interact with tangible cultural resources and enhance their understanding of and affinity with them. Panoramic VR [18-21] navigation is now widely used in many areas, especially after Facebook, YouTube, Twitter, and other social networking sites have launched 360-degree panoramic live streaming function; the application of scenic VR is more popular among users. The VR is designed based on the realistic environment, allowing users to use their own perspective to navigate independently and generate an immersive experience, hence, the highest level of realism and interactivity. By combining the new form of virtual tourism with real-world attractions through information systems development, network transmission, and panoramic VR technologies, travellers can utilize these integrated technologies over the Internet for pretrip preparation and itinerary planning of target destinations.

\section{Methods}

The Delphi method [24] is being increasingly used in investigation of variety of local, regional, and global issues among stakeholders in which a consensus is to be conducted [25-27]. Some of these areas included the development of information systems, and this method is also proven a popular tool to extract the unbiased information for a panel of experts [28-31]. Therefore, it would be appropriate to adopt the Delphi method for obtaining a set of major dimensions and indicators established for the target touring mobile information system from the expert opinions. In the Delphi method, a series of condensed questionnaires is delivered to a group of experts, and their expert opinions are thus collected and examined to establish reliable technologies at a consensus. Here, several rounds of questionnaire responses were collected in an anonymous and non-face-to-face manner. After all questionnaire responses were investigated, the results along with new questionnaires were distributed to the experts as the reference for revising prior opinions. This process was repeated until disagreement among the experts was minimized and a concrete consensus was formed. The common advantages of the Delphi method are that the group composed of experts with various backgrounds can discuss a given topic from diverse perspectives and thereby extend the width of conclusions. Through the statistical data and opinions collected from the multiple rounds of questionnaire responses, a set of indicators was established for the expert's consensus initiatives.

3.1. Delphi Procedure. The Delphi method adopted in this research consisted of two rounds. In the first stage, 10 experts of relevant domains and objective perspectives were invited to provide their opinions through the Delphi questionnaire surveys. These experts could express their opinions freely. The aim of this stage was to enable brainstorming among the experts, rather than posing restriction on the experts' scope of thinking. When an expert found any dimension or indicator listed in the questionnaire insufficient or inappropriate, the expert was asked to provide their additional comments and suggestions. The questionnaire comprised three main dimensions and nine indicators. The experts were asked to rate the importance and feasibility of each indicator according to a 5-point Likert scale, with anchors of 1 (strongly disagree) and 5 (strongly agree) [32]. After the first round of questionnaire, we adopted content analysis to classify expert opinions, after which they developed the second-round questionnaire. Higher scores represented greater importance and practical feasibility of VR development for temple tour guiding.

3.2. Criterion Measure. In this research, we adopt some evaluation criteria $[26,33-36]$ to rank the importance of each of the component. This measurement includes the mean value $(M)$, the standard deviation (SD), and the quartile deviation (QD). Furthermore, to assess agreement among experts, the stopping criteria are measured by using the coefficient of variation (CV) and Kendall's coefficient of concordance (W) [37]. Therefore, the comparison criteria are described as follows:

Suppose that indictor $j$ is given the rating $\mathbf{R}_{\mathbf{i j}}$ judged by the expert $i$, where there are in total $n$ indictors and $m$ experts' judges.

(1) The mean value $(\mathbf{M})$ of the total rating given to indictor $j$ is defined as 


$$
\mathbf{M}_{j}=\frac{1}{m} \sum_{i=1}^{m} \mathbf{R}_{\mathrm{ij}}
$$

(2) The standard deviation (SD) of the total rating given to indictor $j$ is defined as

$$
\mathbf{S D}_{j}=\sqrt{\frac{1}{m} \sum_{i=1}^{m}\left(\mathbf{R}_{\mathbf{i j}}-\mathbf{M}_{\mathbf{j}}\right)^{2}} .
$$

(3) The quartile deviation (QD) of the total rating given to indictor $j$ is defined as

$$
\mathbf{Q D}_{j}=\frac{\left(\mathrm{Q} 3_{j}-\mathrm{Q} 1_{j}\right)}{2},
$$

where $Q 1{ }_{j}$ and $Q 3{ }_{j}$ represent the 1st quartile and the 3 rd quartile of the indictor $j$, respectively.

(4) The coefficient of variation (CV) of the total rating given to indictor $j$ is defined as

$$
\mathbf{C V}_{j}=\frac{\mathbf{S D}_{j}}{\mathbf{M}_{j}}
$$

(5) Kendall's coefficient of concordance (W) $[14,18]$ of the total rating is defined as

$$
\mathbf{W}=\frac{12 S}{\left(m^{2}\left(n^{3}-n\right)-m T\right)},
$$

where the sum of squared deviations, $S$, is defined as

$$
\begin{aligned}
& R_{i}=\sum_{j=1}^{m} \mathbf{R}_{\mathrm{ij}}, \\
& \bar{R}=\frac{1}{n} \sum_{i=1}^{n} R_{i}, \\
& S=\sum_{i=1}^{n}\left(R_{i}-\bar{R}\right)^{2} .
\end{aligned}
$$

$T$ is a correction factor for tired rating:

$$
\begin{aligned}
T & =\sum_{j=1}^{m} T_{j}, \\
T_{j} & =\sum_{i=1}^{g_{j}}\left(t_{i}^{3}-t_{i}\right),
\end{aligned}
$$

in which $t_{i}$ is the number of tied rating in the $i$ th group having the same constant value of tied rating and $g_{j}$ is the number of groups of ties in the set of rating for expert $j$.

3.3. Consensus Criteria. To obtain consensual and important opinions from a group of experts in this study, the major statistics used in the Delphi method are a measure of central tendency (M), level of dispersion (SD and QD), and level of agreement and stability ( $\mathbf{C V}$ and $\mathbf{W}$ ). In general, the use of mean value to represent the central tendency is favoured [26-30]. Table 1 shows the level of importance based on the 5 -point Likert scale. An indictor's mean score of more than 4.2 ("very high important"), or more than 3.5 and less than or equal 4.2 ("high important") is recommended in the first round of Delphi procedure. A higher mean value indicates more importance of the critical factor. Furthermore, after justifying the mean value, standard deviations and quartile deviations are then identified to measure the amount of variation or dispersion of items. Table 2 shows the level of variation or dispersion according to SD and QD. In this study, the dispersion level is divided into three levels (high, moderate, and no consensus). The dispersion level is accepted as high if $\mathbf{S D}$ is less than or equal to 1 and $\mathbf{Q D}$ is less than or equal to $0.5[38,39]$. A low deviation indicates that the values tend to be close to the mean value (M), while a high deviation indicates that the values are spread out over a wider range. Moreover, we adopt the coefficient of variation $(\mathbf{C V})$ and Kendall's coefficient of concordance $(\mathbf{W})$ to determine whether a consensus has been met or not. It is an important issue to know when to stop the Delphi procedure. If the procedure is finished too early, the results may not be significant; and, if the process has too many rounds, the task may be too heavy to the experts and consequently contribute to withdrawals [38-40]. Table 3 shows the level of consensus determined by $\mathbf{C V}$ and $\mathbf{W}$. For example, the level of consensus value $(0.8<\mathbf{C V}$ and $0 \leq \mathbf{W} \leq 0.3)$ shows a weak level of agreement of the experts' opinion, but still needs for additional round.

3.4. Analysis of Results. The questionnaire copies were distributed to 10 experts, who were willing to assist with the study. Before the experts filled in the questionnaire, they were asked to carefully read the instructions for responding to the questionnaire. The questionnaire responses were collected 2 weeks after distribution. The response rate was $100 \%$. The questionnaire comprised three main dimensions and nine indicators, as shown in the first column of Table 4. Furthermore, consensus was reached on 2 out of 9 indicators, but there were two indicators did not achieve consensus. These include reminder function $(\mathbf{M}=2.7 \leq 3.5)$ and rmart terminal management $(\mathbf{M}=3.0 \leq 3.5$; $Q \mathbf{Q D}>0.5)$; thus, these two indicators were removed in the next round. Moreover, W was computed to estimate the level of agreement and consensus among experts in the first round. The result $(\mathbf{W}=0.3 \leq 0.3)$ reveals a weak agreement and need for additional round.

The statistical analysis results of the first-round questionnaire responses revealed that the mean values of "reminder function" and "smart terminal management" were smaller than 3.5 and were thus deleted. Similarly, the second-round questionnaire copies were distributed to all experts who completed the first-round questionnaire. In total, 10 copies were distributed, and the response rate was $100 \%$. Table 5 shows that all SD values are smaller than 1 , QD and CV values are also equal or smaller than 0.5 , and Kendall's W reaches a high level of consensus 
TABLE 1: Level of importance.

\begin{tabular}{lr}
\hline Mean $(\mathbf{M})$ & Level of importance \\
\hline $4.2<\mathbf{M} \leq 5$ & Very high important \\
$3.5<\mathbf{M} \leq 4.2$ & High important \\
$2.7<\mathbf{M} \leq 3.5$ & Moderate important \\
$\mathbf{M} \leq 2.7$ & Low important \\
\hline
\end{tabular}

TABLE 2: Level of dispersion.

\begin{tabular}{lcc}
\hline Standard deviation $(\mathbf{S D})$ & Quartile deviation $(\mathbf{Q D})$ & Level of dispersion \\
\hline $0 \leq \mathbf{S D} \leq 1$ & $0 \leq \mathbf{Q D} \leq 0.5$ & High \\
$1<\mathbf{S D} \leq 1.5$ & $0.5<\mathbf{Q D} \leq 1$ & Moderate \\
$1.5<\mathbf{S D}$ & $1<\mathbf{Q D}$ & Low and no consensus \\
\hline
\end{tabular}

TABLE 3: Level of agreement and consensus.

\begin{tabular}{lcc}
\hline Coefficient of variation $(\mathbf{C V})$ & Kendall's coefficient of concordance $(\mathbf{W})$ & Level of agreement and stability \\
\hline $0 \leq \mathbf{C V} \leq 0.5$ & $0.5 \leq \mathbf{W}$ & Good agreement, no additional round \\
$0.5<\mathbf{C V} \leq 0.8$ & $0.3<\mathbf{W} \leq 0.5$ & Moderate agreement, possible need for another round \\
$0.8<\mathbf{C V}$ & $0 \leq \mathbf{W} \leq 0.3$ & Weak agreement, need for additional round \\
\hline
\end{tabular}

TABle 4: Results for the Delphi first round.

\begin{tabular}{|c|c|c|c|c|}
\hline Dimension/indicator & $\mathbf{M}$ & SD & QD & $\mathrm{CV}$ \\
\hline \multicolumn{5}{|l|}{ Functional perspective } \\
\hline Search function & 4.5 & 0.67 & 0.50 & 0.15 \\
\hline Feedback function & 4.5 & 0.67 & 0.50 & 0.15 \\
\hline Reminder function & 2.7 & 0.78 & 0.5 & 0.29 \\
\hline \multicolumn{5}{|l|}{ Technology integration } \\
\hline Smart terminal management & 3.0 & 0.77 & 0.75 & 0.26 \\
\hline Social media integration & 4.7 & 0.64 & 0.25 & 0.14 \\
\hline APP development & 3.6 & 0.68 & 0.50 & 0.19 \\
\hline \multicolumn{5}{|l|}{ Smart system } \\
\hline Tour guiding system & 4.7 & 0.64 & 0.25 & 0.14 \\
\hline Navigation system & 3.7 & 0.64 & 0.50 & 0.17 \\
\hline Archival system & 3.9 & 0.70 & 0.50 & 0.18 \\
\hline \multicolumn{5}{|c|}{ Kendall's $\mathbf{W}=0.30$} \\
\hline
\end{tabular}

$(\mathbf{W}=0.78 \geq 0.5)$. These results reveal a better agreement and consensus on the responses in this round. At this stage, expert opinions had reached consensus; therefore, the third round of the questionnaire was considered unnecessary. The importance and feasibility of the indicators were evaluated according to a 5-point Likert scale. The importance and feasibility options were scored according to the expert opinions. The mean values of "navigation system" $(\mathbf{M}=3.9)$ were between 3.4 and 4.2 , and those of the remaining indicators were larger than 4.2. All these indicators were ranked as "very high important." Higher scores represent greater importance and practical feasibility of VR development for temple tour guiding.

\section{VR Touring System Framework and Development}

In this study, we use the Delphi method by the experience and intuition of experts to integrate the criteria for finding the functionalities of the interactive VR touring system accurately. Moreover, according to Table 5, the system functionalities and system architecture are then established. This section provides an overview of the system functionalities and architecture. Finally, the A-Frame framework and panoramic VR construction process are also described.

4.1. System Functionalities. The local culture of Taiwan is conveyed through the system that allows the faithful and tourists to understand the history of the place. Therefore, after collecting in-depth information on the historical background and cultural references of the Jian Temple as well as visiting the site and interviewing the visitors, we found out that the general users would like to use the system to achieve the following purposes:

(1) Planning and design of a site-specific guided tour to provide visitors with an understanding. As temples are interacting with people and are constantly being updated in the flow of time and space, the temples themselves are showcases that allow visitors to learn 
and experience culture in a way that is not only spiritual but also subliminal. VR allows users to get to know the environment of the temple and the order and location of the rituals so that they can learn about it without being there.

(2) By completing the planning of the guided tours, the temple grounds can become an exhibition space, which will not only attract visitors but also bring in crowds and give a different impression and experience to religious tourism. The VR application also allows users to learn about the history of the temple and the stories of the deities without ever visiting the site.

Therefore, we have initially summarized the three major dimensions and nine indicators for the actual construction of the virtual temple space in the form of an interactive tour and VR technology, and through the Delphi method, an expert group decision-making technique, we have implemented two rounds of questionnaires and conducted a round-robin feedback process to integrate the opinions of various experts, from which we have selected the important indicators for the application of VR in interactive tours, which have been agreed upon by the experts, as a reference for the introduction of the tour guiding system at the shrine. In addition, this study focuses on user interaction for system development, and under the framework of relevant software development methods, the user guide system is designed to be implemented on the temple site so that the system becomes an important medium for human interaction with the environment, allowing tourists to adopt a self-guided approach to stimulate their experience of traditional culture and add value to the guiding. After two less important and significant indicators were eliminated according to Table 5 of Delphi assessment processes, the requirement of the VR touring system was established. The VR system mainly demonstrated the temple space through interactive guiding and VR technologies. The goal of the system is to design VRbased interaction different from conventional tour guiding methods to help users view the display space from their points of view. The users cannot only control camera view angles but also select the paths and guiding information as if they were actually walking in the temple. Considering the results of the Delphi assessment, we propose the interactive VR touring system should be the next step in design modifications and a basis for future development. This VR touring system is divided into UI framework subsystem, VR guide subsystem, mobile APP subsystem, and archival subsystem, as depicted in Figure 2.

4.2. System Framework and Architecture. To be able to quickly prototype and evaluate VR touring experience, we focus on the web-based backend system as target application platform to meet the requirements specified in Section 4.1. Smart devices are usually equipped with a camera as well as mobile positioning and wireless Internet access; this study employed these features in a cross-platform VR mobile application with multimedia and LBS capabilities. The system architecture comprises three major parts: the user, frontend, and backend views, as depicted in Figure 3. Moreover, these multimedia capabilities are supported a broad range of web-based application on many mobile device and not tied to specific wearable device/VR glasses or PC/Laptop. In this study, WebVR [41, 42] was built to present the virtual temple space with interactive guidance and VR technology so that users can browse the exhibition space from their own viewpoint and not only can they control the camera angle but also choose their own browsing path and navigation information, making them feel like they are actually in the scene. Finally, we used the Cordova framework to achieve cross-platform results and to reduce the problems caused by the difference in platforms among users.

4.2.1. Frontend of System Architecture. A-Frame [43] API is then used to construct WebVR effects. A-Frame is an opensource framework for the Three.js physical component system for VR experiences. Developers can create 3D and WebVR scenes using HTML, while incorporating popular game development patterns used by engines such as Unity. This technique offers a complete immersive and interactive experience including 360-degree content, interactive effects that support location tracking and control of the device, and cross-platform device usage such as VR glasses headmounted displays, smartphones, and computer desktops. Furthermore, Primitives [43] in A-Frame Docs features basic geometric shapes (boxes, spheres, cylinders, planes, etc.), Collada models that can be created by importing $3 \mathrm{D}$ modelling tools or downloaded from the Internet, a sky that defines the background, and a camera that defines the angle from which the user views the scene, animation, light and shadow, panoramic video, etc. Through HTML tags, developers can easily create WebVR scenes. Thus, the VR tour guiding system built using A-Frame enabled the users to view the webpages on smart devices anytime and anywhere. With the immediate display on webpages, the applications of VR become increasingly convenient and improve users' visual experience. Moreover, the website system was presented in conventional text and graphics modes combined with fancy effects created using jQuery. Javascript was used to resolve problems between different browsers to present latest news and the most comfortable viewing experience to users. Social media were used to introduce the Ji'an Temple and create a brand on the social media platform to increase the popularity of the temple. The Facebook fan page of the temple attracts more than 2000 users. The manager can analyze and optimize relevant data through Google Analytics and FB data to evaluate whether posts achieve desired effects.

4.2.2. Backend of System Architecture. The main computer runs a Node.js server, and the server and its database are first established to provide VR mobile tour services. Relevant service elements are compiled to perform background services by rendering the VR scene with A-Frame. Through such interaction, the user can acquire information about an 
TABle 5: Results for the Delphi second round.

\begin{tabular}{|c|c|c|c|c|}
\hline Dimension/indicator & $\mathbf{M}$ & SD & QD & $\mathrm{CV}$ \\
\hline \multicolumn{5}{|l|}{ Functional perspective } \\
\hline Search function & 4.8 & 0.42 & 0.25 & 0.09 \\
\hline Feedback function & 4.6 & 0.52 & 0.5 & 0.11 \\
\hline \multicolumn{5}{|l|}{ Technology integration } \\
\hline Social media integration & 4.7 & 0.48 & 0.5 & 0.10 \\
\hline APP development & 4.5 & 0.71 & 0.5 & 0.16 \\
\hline \multicolumn{5}{|l|}{ Smart system } \\
\hline Tour guiding system & 4.8 & 0.42 & 025 & 0.09 \\
\hline Navigation system & 3.9 & 0.57 & 0.25 & 0.15 \\
\hline Archival system & 4.8 & 0.42 & 0.25 & 0.09 \\
\hline \multicolumn{5}{|c|}{ Kendall's W $=0.78$} \\
\hline
\end{tabular}

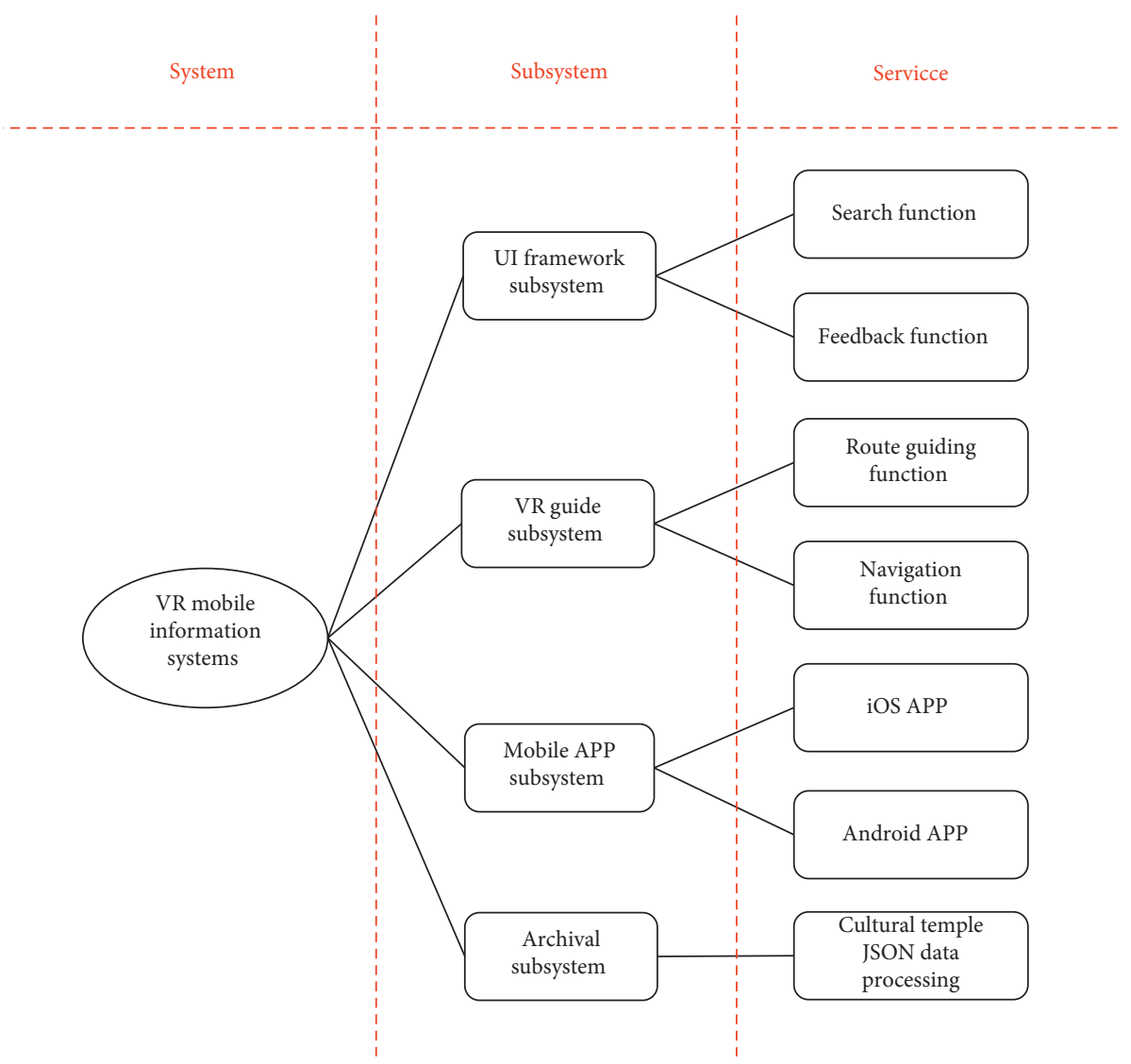

FIGURE 2: System functionalities.

attraction. In this manner, both modes integrate virtual images into a real-world setting, providing an interactive guided tour to users of the application. The Cordova plugin [44] is used to establish the cross-platform VR mobile tour system's backend data interface, which is connected and synchronized with the server and database. Cordova is an open-source mobile application development framework designed to enable programmers to develop cross-platform mobile applications with web-based application programming interfaces (APIs). It also grants JavaScript access to these interfaces to gain control of system hardware resources (e.g., camera or compass). Because smart devices run on numerous operating systems, Cordova can be used to help programmers ensure that uniform results are achieved across various platforms, thus minimizing the problems users face when changing platforms. Cascading style sheets are used to organize the screen layout and control the background opacity. The final results are transmitted to a browser (HTML format).

\subsubsection{Some Discussion on the Panoramic VR Construction} Process. The use of virtual reality devices is becoming more and more popular, and with the increase in demand, developers are more actively betting on this. However, there is currently no standard specification for the service content of 


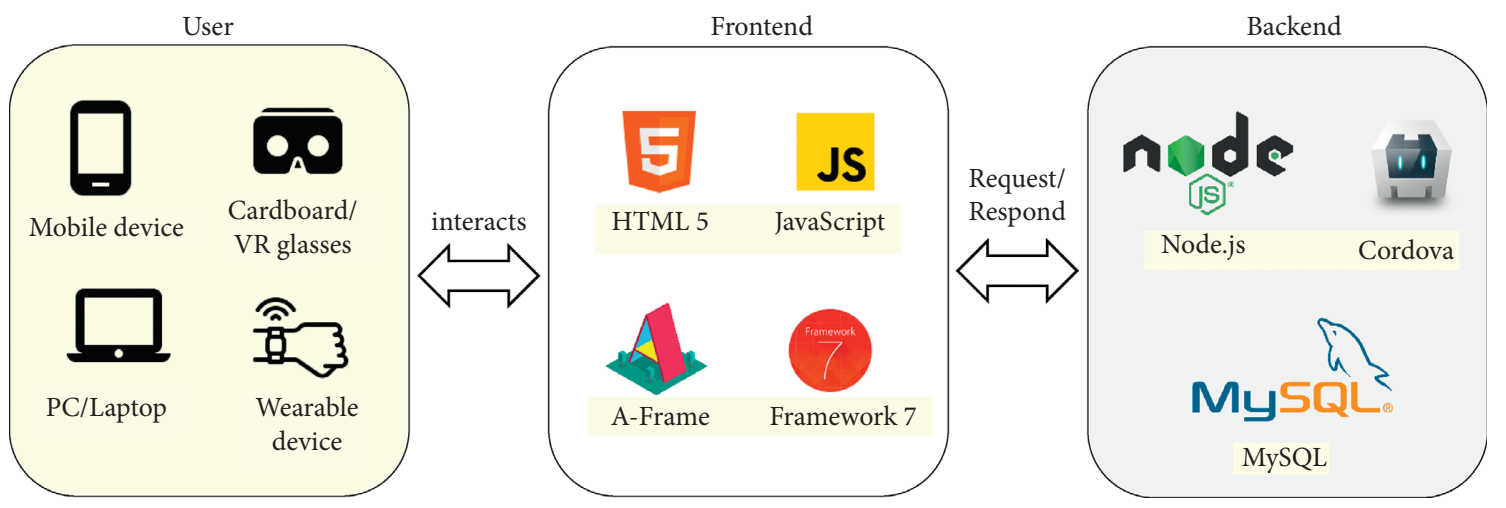

FIGURE 3: System architecture.

virtual reality; therefore, it is difficult for all users to achieve the best experience with the application of virtual reality. When the real-time update of a program is too slow, the screen resolution is too low, or the design does not consider the body feel and the environment, and it may cause discomfort to the user. This research has developed a VR system that can be applied to any device using only a web link. The previous VR devices are expensive, and each VR application usually has its own dedicated device, and the application programs of the devices are not in common with each other. Even if most people are interested in VR, they cannot afford the cost of enjoying VR. The development of WebVR is to solve the current bottleneck of VR promotion. To use WebVR technology, there is no need to learn new programming languages or new VR development software packages from previous VR developers. WebVR allows developers to quickly get started with the VR development model through web languages such as JavaScript and HTML. As in the past, you need to spend a lot of money to buy professional software. A simple web editor can immediately join the developer's team, which greatly reduces the entry barrier for VR development. Furthermore, through the open-source A-Frame framework, VR scenes can be used on mobile phones, PC, Oculus Rift, and HTC Vive. Using this construction method has the following advantages and characteristics: (1) A-Frame can reduce redundant codes. Redundant and complex code has become an obstacle for early adopters. A-Frame reduces the complex and redundant code to one line of HTML code. For example, only one <ascene $>$ tag is required to create a scene. (2) A-Frame is specially designed for web developers. It is based on DOM, so it can manipulate 3D/VR content like other web applications. Of course, it can also be used in conjunction with JavaScript frameworks such as React; (3) A-Frame makes the code structured. The code of Three.js is usually loose. A-Frame builds a declarative entity-component-system on top of Three.js. In addition, the components can be published and shared so that other developers can use them in the form of HTML.

Therefore, to provide users with a deeper understanding of the culture and sights of Ji'an Temple, VR display technology was employed in this project, with the aim of superimposing real-life images and data with computer- generated virtual objects to create a complex visual sensory experience and increase users' knowledge of the area. However, to familiarize users with the local environment, we would design interactive and immersive VR tours with panoramic photos/videos of the area to enable users to learn about the culture and characteristics of the locality through the game and to increase their willingness to participate in the interaction, while the designers were able to add more tourist information to the existing tourist environment, thus increasing the feasibility of navigation. The following describes the steps and research methods required to build a panoramic VR navigation system, as shown in Figure 4. The first step is to use a panoramic camera to photograph the environment of the tour location shown in Figure 5 and dynamically load the JSON formatted scenery tour information to the server. The panoramic photographs/videos shot are imported into the A-Frame framework as a graphical scene builder, scripted for control and other related functions such as viewing angles, and developed for use in panoramic VR navigation via Cordova cross-platform.

4.3. System Demonstration. The VR tour guiding system built using A-Frame enabled the users to view WebVR on smart devices anytime and anywhere. With the immediate display on webpages, the applications of VR became increasingly convenient and improve users' visual experience. The website was presented in conventional text and graphics modes combined with fancy effects created using jQuery. Javascript was used to resolve problems between different browsers to present latest news and the most comfortable viewing experience to users. A-Frame was used to create a VR system. In the VR system of Ji'an Temple tour guiding, a user can see a trigger spot in the center of the monitor (Figure 6). Users can swipe the screen of cell phone or move their cell phone to select a position they want to go. Clicking a position can trigger corresponding locations. Figure 7 demonstrates that films containing information of relevant cultural relics are available and audio guiding that can be played by clicking the play button below. In addition, social media are used to introduce Ji' an Temple and create a brand on the social media platform to increase the popularity of the temple. The manager of the fan page sometimes 


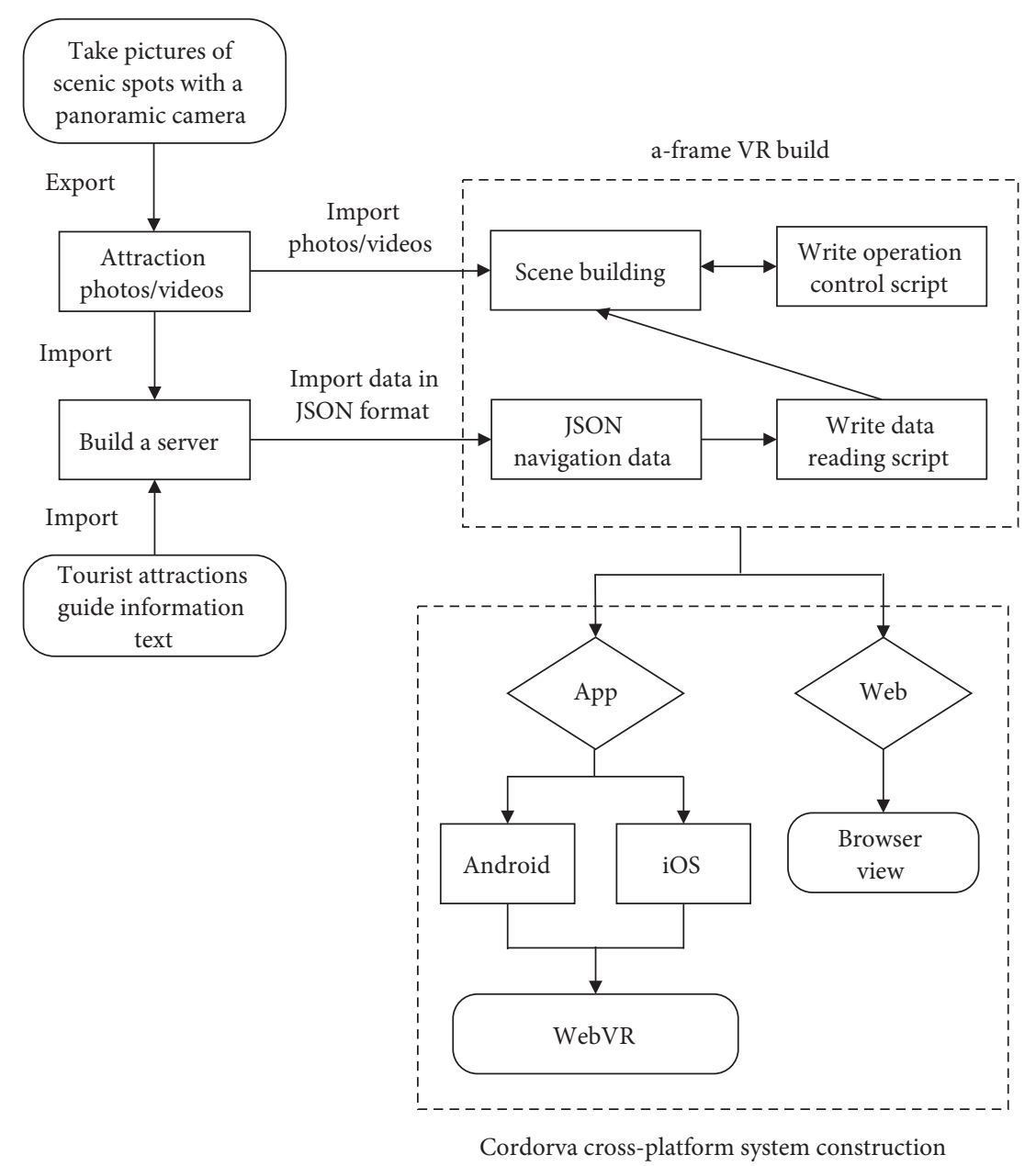

FIgURE 4: The workflow for building a panoramic VR touring system.

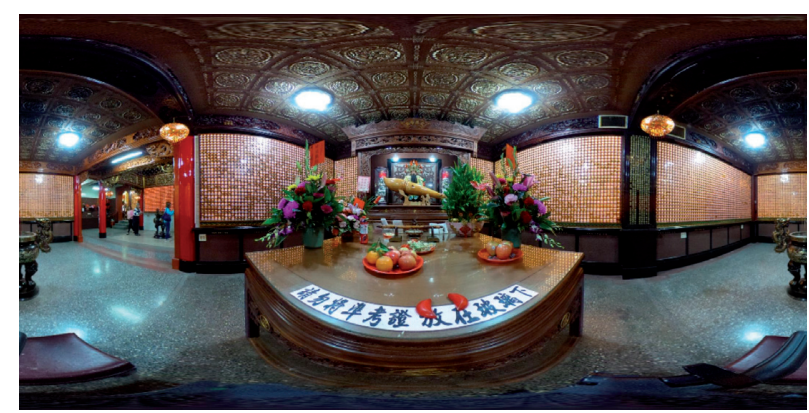

Figure 5: An example of the panoramic image in Jian Temple.

plan and design graphics and text contents of posts, and he can also analyze and optimize relevant data through Google Analytics and FB data to evaluate whether posts achieve desired effects. Finally, the display of operation of VR headset glasses is shown in Figure 8.

\section{QUIS Results and Discussion}

To understand the user's satisfaction with the usage of the VR touring system, we employed the Questionnaire for User Interaction Satisfaction (QUIS) [45] to conduct a quantitative study. The survey respondents are mainly temple officials, pilgrims, and Internet citizens. Moreover, the main focus was on answering representative questions with adequate satisfaction and helpfulness. The questions were answered by users following their action learning using the VR touring system. The questionnaire had four dimensions: (1) overall reaction to the VR touring system; (2) display of the VR touring system; (3) contents of the VR touring system; and, (4) usage of the VR touring system. The questionnaire is divided into these four parts and contains 18 questions. Among them, the users were asked to rate the importance and feasibility of each 


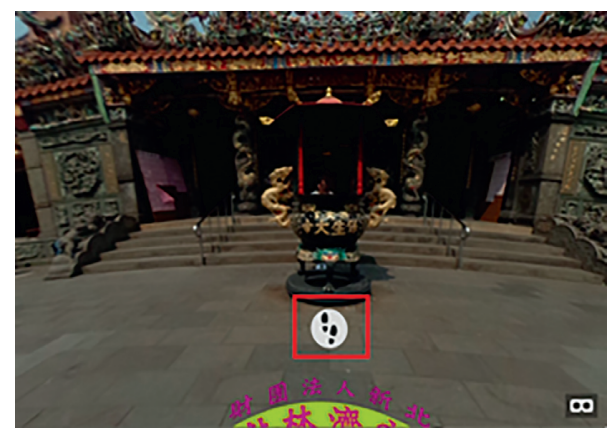

Figure 6: Independent control of tour routes.

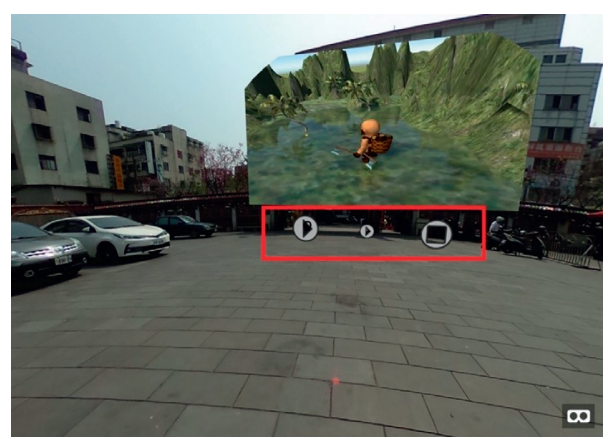

Figure 7: Video story and audio guiding.

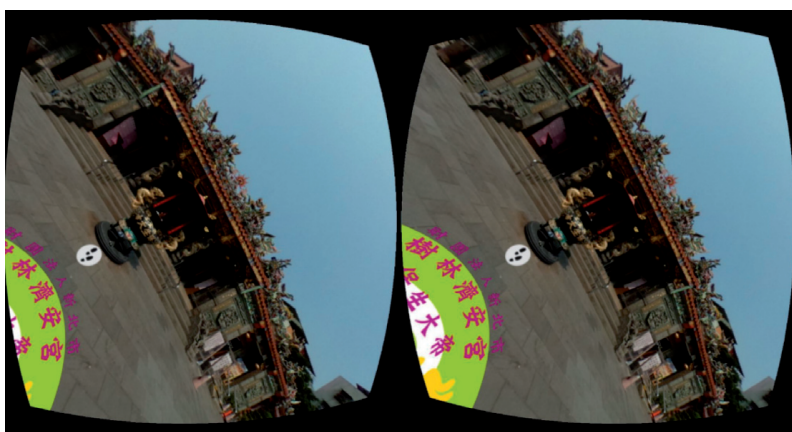

Figure 8: Display of operation of VR headset glasses.

indicator according to a 6-point Likert scale, with anchors of 1 (strongly disagree) and 6 (strongly agree) [32]. The aim was to obtain a general understanding of the satisfaction of each user, which could provide evidence and a foundation for data analyses. From Table 6, the mean values of the four dimensions revealed that the usage of the VR touring system as favorable (4.72); however, their overall satisfaction toward the tour guiding display was slightly lower (4.53). In a further analysis of various items, the median value of 3.5 was used for a 6-point Likert scale. The mean values of 18 items were higher than 3.5, the median value, suggesting that the users generally considered the VR touring system to be satisfactory.
The mean values of the first item "I think the VR touring system is (undesirable/excellent)" and the second item "I think the VR touring system is (difficult to use/easy to use)" were close to 5 points, indicating that the proposed system was easy to operate and excellent in quality. However, the mean values of the sixth item "I think the VR touring system is (dull/lively)" and the ninth item "The resolution clarity of the screen is (blurred/clear)" were 4.00 and 4.17 , respectively, that is, the sixth item and the ninth item are the two poorer satisfactions. These results revealed that this system is generally satisfactory and easy to operate, but relatively uninteresting. 
TABLE 6: QUIS result analysis.

\begin{tabular}{|c|c|c|c|}
\hline Items & $\begin{array}{c}\text { Rating } \\
123456 \\
\end{array}$ & $\mathbf{M}$ & SD \\
\hline Overall reaction to the VR touring system & & 4.53 & \\
\hline I think the VR touring system is: & Undesirable/excellent & 5.00 & 0.739 \\
\hline I think the VR touring system is: & Difficult to use/easy to use & 5.00 & 0.426 \\
\hline I think the VR touring system is: & Unsatisfactory/satisfactory & 4.50 & 1.087 \\
\hline \multicolumn{4}{|l|}{ I think the performance of the VR touring } \\
\hline System is: & Low/high & 4.42 & 0.669 \\
\hline I think the VR touring system is: & Boring/exciting & 4.25 & 0.622 \\
\hline I think the VR touring system is: & Dull/lively & 4.00 & 1.044 \\
\hline Display of the VR touring system & & 4.61 & \\
\hline To read the information on the screen is: & Difficult/easy & 4.83 & 0.835 \\
\hline The structure of system functionalities is: & Confusing/obvious & 4.83 & 0.835 \\
\hline The resolution clarity of the screen is: & Blurred/clear & 4.17 & 0.718 \\
\hline Contents of the VR touring system & & 4.67 & \\
\hline \multicolumn{4}{|l|}{ The relevance of touring contents and usage } \\
\hline Requirements is & Never/always & 4.67 & 0.778 \\
\hline The position of the message on the screen is: & Inconsistent/consistent & 4.67 & 0.888 \\
\hline Does the touring help to further understand? & Never/always & 4.75 & 0.622 \\
\hline Usage of the VR touring system & & 4.72 & \\
\hline To self-operate the VR touring system is: & Difficult/easy & 4.83 & 0.937 \\
\hline To use the mobile tracking function is: & Difficult/easy & 4.42 & 0.996 \\
\hline \multicolumn{4}{|l|}{ To use the audio and video touring } \\
\hline Functions is: & Difficult/easy & 4.92 & 0.996 \\
\hline Compared with the text navigation, is & & & \\
\hline the VR touring system easier to understand? & Never/always & 4.58 & 0.793 \\
\hline The prompt message displayed on the screen is: & Helpless/helpful & 4.83 & 1.115 \\
\hline
\end{tabular}

\section{Conclusions}

The system evaluation was divided into two stages: expert assessment and target user usability test. In the first stage, 10 experts of relevant domains and objective perspectives were invited to provide their opinions through the Delphi questionnaire surveys. Through literature review, we summarized three major dimensions and nine indicators for the experts to evaluate. Subsequently, based on the experts' opinions, two indicators (reminder function and smart terminal management) were removed. VR tour guiding was established thereafter. In the second stage, users, including students, Internet users, and pilgrims, were invited. VR tour guiding, based on the integrated outcomes of the Delphi expert questionnaires, was provided to these participants, and the usability of the system was tested. QUIS was employed to analyze the users' satisfaction to the tour guiding system; the satisfaction analysis comprised four main axes: the overall reaction, tour guiding display, tour guiding content, and tour guiding operation. The developed system was rated by the target users through the QUIS for its usability. This study analyzed the overall reaction to the tourguiding system, display, content, and operation. The analysis results revealed that, in general, the users perceived VR tour guiding to be convenient and easy to use. The tour guiding system's display and content presented clear information to the users, aiding them in gaining further understanding of the introduced item. Statistical analysis results of the expert and user questionnaires revealed that interactive VR was more efficient in communicating information to users than conventional text and graphics webpages. Because interactive VR tour guiding was incorporated into the proposed system, the interaction between the users and objects increased the users' concentration as well as information communication efficiency. Through this combination, a revolutionary method of tour guiding was developed. Conventional graphics and text interpretation can no longer satisfy the needs of the general public. Additional information technologies must be integrated with tour guiding to attract user attention by adding diverse options to interactive VR tour guiding systems. The easy-to-operate and convenient systems can provide unprecedented online browsing experience to users or customers of businesses and subsequently strengthen customers' attachment to businesses effectively.

\section{Limitations and Future Studies}

There is still room for improvement in this research in the future. First of all, our target sample is limited to the case of Ji'an Temple in Taiwan. It did not take into account the technological and cultural changes between different countries, so a limitation with our chosen method was that we were unable to make any detailed comparisons between application performance and user experience with other methods. Only QUIS research can be conducted. Furthermore, this research uses a panoramic image method to implement a VR navigation system. Although it has a three- 
dimensional effect, the spherical virtual image will form an obvious intersection point stitching problem directly above and directly below the space. In the future, it is hoped that the problem of the intersection of the spheres can be improved without affecting the three-dimensional effect. In addition, at present, the concept of simulating the horizontal distance between the eyes is used to draw the scene horizontally. If you turn your head in other directions to watch, it will be visually distorted and the images will not be able to merge. In the future, further discussions can be made in this direction, which is expected to reduce the dizziness. Finally, the future application of VR combined with imagination can develop infinite possibilities; in addition to the existing visual and auditory senses, humans still have touch, smell, taste, and various other senses so that users can have a more immersive experience in the virtual world. Integrating these perceptions in the future will be one of the important directions. Therefore, including how to simulate various perceptions, how to combine, transmit, and synchronize various sensory signals, etc., are all important topics; in addition, new applications developed by VR, such as how to remotely control through VR and how to provide further $360^{\circ}$ panoramic video services with lower latency will also drive the evolution and development of various new technologies.

\section{Data Availability}

The data used to support the findings of the study are included within the article.

\section{Disclosure}

Part of the results of this research is also published at the 3rd IEEE International Conference on Knowledge Innovation and Invention (https://ieeexplore.ieee.org/document/9318862).

\section{Conflicts of Interest}

The authors declare that they have no conflicts of interest regarding the publication of this paper.

\section{Acknowledgments}

This research was partially supported by the "Teaching Practice and Research Program" (PEE1080223), sponsored by the Ministry of Education and the Ministry of Science and Technology, Taiwan, R.O.C., under Grant MOST 108-2221E-431-003.

\section{References}

[1] Y. Xie, L. Ryder, and Y. Chen, "Using interactive virtual reality tools in an advanced Chinese language class: a case study," TechTrends, vol. 63, no. 3, pp. 251-259, 2019.

[2] H.-M. Chiao, Y.-L. Chen, and W.-H. Huang, "Examining the usability of an online virtual tour-guiding platform for cultural tourism education," Journal of Hospitality, Leisure, Sports and Tourism Education, vol. 23, pp. 29-38, 2018.

[3] B. O. Han, Y. H. Kim, K. Cho, and H. S. Yang, "Museum tour guide robot with augmented reality," in Proceedings of the 16th International Conference on Virtual Systems and Multimedia (VSMM), pp. 223-229, IEEE, Seoul, Korea, 2010.

[4] D. M. Zhou, D. Shi, B. Wang et al., "Research on virtual tourist guide training system based on virtual reality technology," in Proceedings of the Third International Symposium on Knowledge Acquisition and Modeling, pp. 155-158, IEEE, Wuhan, China, 2010.

[5] J. H. Lo and G. Z. Gong, "Touring system using augmented reality-A case study of yilan cultural industries," in Proceedings of the 3rd IEEE International Conference on Knowledge Innovation and Invention (ICKII), pp. 204-207, IEEE, Kaohsiung, Taiwan, 2020.

[6] Y. T. Hsu, "Recognition, designation, and local Practice of "folk custom and related cultural artifacts:" an example of "Yunlin Liu Fang Ma Guolu"” Min Su Qu Yi, vol. 193, pp. 221-265, 2016.

[7] P. R. Katz, "Bridging the gaps: methodological challenges in the study of Taiwanese popular religion," International Journal of Taiwan Studies, vol. 1, no. 1, pp. 36-63, 2018.

[8] Shulin Ji'an Temple, 2021, https://www.jian.org.tw, In Chinese.

[9] K. W. Hsieh, "A review and analysis of the studies of the Baosheng emperor belief," Journal of World Religions, vol. 16, pp. 95-124, 2010, In Chinese.

[10] J. H. Lo and M. J. You, "Design and implementation of the interactive virtual reality touring system-a case study of Shulin Ji'an temple in Taiwan," in Proceedings of the 3rd IEEE International Conference on Knowledge Innovation and Invention (ICKII), pp. 115-117, IEEE, Kaohsiung, Taiwan, 2020.

[11] Gartner's Top 10 Strategic Technology Trends for 2020, 2021, https:/www.gartner.com/smarterwithgartner/gartner-top10-strategic-technology-trends-for-2020/.

[12] A. Dix, A. J. Dix, J. Finlay, G. D. Abowd, and R. Beale, HumanComputer Interaction, Pearson Education, London, UK, 2004.

[13] K. Choi, Y.-J. Yoon, O.-Y. Song, and S.-M. Choi, "Interactive and immersive learning using $360^{\circ}$ virtual reality contents on mobile platforms," Mobile Information Systems, vol. 2018, Article ID 2306031, 12 pages, 2018.

[14] H. Huang and C.-F. Lee, "Factors affecting usability of 3D model learning in a virtual reality environment," Interactive Learning Environments, pp. 1-14, 2019.

[15] J. Steuer, "Defining virtual reality: dimensions determining telepresence," Journal of Communication, vol. 42, no. 4, pp. 73-93, 1992.

[16] Y. Chang and G.-P. Wang, "A review on image-based rendering," Virtual Reality \& Intelligent Hardware, vol. 1, no. 1, pp. 39-54, 2019.

[17] J. H. Lee and C. E. Rhee, "Flexibly connectable light field system for free view exploration," IEEE Transactions on Multimedia, vol. 22, no. 4, pp. 980-991, 2019.

[18] D. Song, T. Li, W. Li, W. Nie, W. Liu, and A. Liu, "Universal cross-domain 3D model retrieval," IEEE Transactions on Multimedia, 2020.

[19] J. Park, I.-B. Jeon, S.-E. Yoon, and W. Woo, "Instant panoramic texture mapping with semantic object matching for large-scale urban scene reproduction," IEEE Transactions on Visualization and Computer Graphics, vol. 27, no. 5, pp. 2746-2756, 2021.

[20] O. Erat, M. Hoell, K. Haubenwallner, C. Pirchheim, and D. Schmalstieg, "Real-time view planning for unstructured lumigraph modeling," IEEE Transactions on Visualization and Computer Graphics, vol. 25, no. 11, pp. 3063-3072, 2019. 
[21] J. Pirker and A. Dengel, "The potential of 360-degree virtual reality videos and real VR for education-A literature review," IEEE Computer Graphics and Applications, 2021.

[22] T. Marrinan and M. E. Papka, "Real-time omnidirectional stereo rendering: generating $360^{\circ}$ surround-view panoramic images for comfortable immersive viewing," IEEE Transactions on Visualization and Computer Graphics, vol. 27, no. 5, pp. 2587-2596, 2021.

[23] H. Shum and S. B. Kang, "Review of image-based rendering techniques," in Visual Communications and Image Processing, vol. 4067, pp. 2-13, International Society for Optics and Photonics, Bellingham, WC, USA, 2000.

[24] H. A. Linstone and M. Turoff, The Delphi Method, AddisonWesley, Reading, MA, USA, 1975.

[25] H. A. Linstone and M. Turoff, "Delphi: a brief look backward and forward," Technological Forecasting and Social Change, vol. 78, no. 9, pp. 1712-1719, 2011.

[26] H. D. Musa, M. R. Yacob, A. M. Abdullah, and M. Y. Ishak, "Delphi method of developing environmental well-being indicators for the evaluation of urban sustainability in Malaysia," Procedia Environmental Sciences, vol. 30, pp. 244-249, 2015.

[27] J. C. Brancheau, B. D. Janz, and J. C. Wetherbe, "Key issues in information systems management: 1994-95 SIM Delphi results," MIS Quarterly, vol. 20, no. 2, pp. 225-242, 1996.

[28] A. P. C. Chan, E. H. K. Yung, P. T. I. Lam, C. M. Tam, and S. O. Cheung, "Application of Delphi method in selection of procurement systems for construction projects," Construction Management and Economics, vol. 19, no. 7, pp. 699-718, 2001.

[29] C. W. Holsapple and K. D. Joshi, "Knowledge manipulation activities: results of a Delphi study," Information \& Management, vol. 39, no. 6, pp. 477-490, 2002.

[30] C. Okoli and S. D. Pawlowski, "The Delphi method as a research tool: an example, design considerations and applications," Information \& Management, vol. 42, no. 1, pp. 15-29, 2004.

[31] R. Schmidt, K. Lyytinen, M. Keil, and P. Cule, "Identifying software project risks: an international Delphi study," Journal of Management Information Systems, vol. 17, no. 4, pp. 5-36, 2001.

[32] A. Joshi, S. Kale, S. Chandel, and D. Pal, "Likert scale: explored and explained," British Journal of Applied Science \& Technology, vol. 7, no. 4, pp. 396-403, 2015.

[33] K. Horner, M. Islam, L. Flygare, K. Tsiklakis, and E. Whaites, "Basic principles for use of dental cone beam computed tomography: consensus guidelines of the European academy of dental and maxillofacial radiology," Dentomaxillofacial $R a$ diology, vol. 38, no. 4, pp. 187-195, 2009.

[34] M. R. Geist, "Using the Delphi method to engage stakeholders: a comparison of two studies," Evaluation and Program Planning, vol. 33, no. 2, pp. 147-154, 2010.

[35] P.-C. Chang, N.-T. Tsou, B. J. C. Yuan, and C.-C. Huang, "Development trends in Taiwan's opto-electronics industry," Technovation, vol. 22, no. 3, pp. 161-173, 2002.

[36] S. Cafiso, A. Di Graziano, and G. Pappalardo, "Using the Delphi method to evaluate opinions of public transport managers on bus safety," Safety Science, vol. 57, pp. 254-263, 2013.

[37] P. Legendre, "Coefficient of concordance," Encyclopedia of Research Design, vol. 1, pp. 164-169, 2010.

[38] J. Henning and H. Jordaan, "Determinants of financial sustainability for farm credit applications-a Delphi study," Sustainability, vol. 8, no. 1, p. 77, 2016.
[39] R. Ab Latif, A. Dahlan, Z. A. Mulud, and M. Z. M. Nor, "The Delphi technique as a method to obtain consensus in health care education research," Education in Medicine Journal, vol. 9, no. 3, 2017.

[40] L. S. Rodrigues and L. Amaral, "Key enterprise architecture value drivers: results of a Delphi study," in Proceedings of the Conference: 21st International Business Information Management Association Conference, IBIMA 2013, Vienna, Austria, 2013.

[41] S. G. Santos and J. C. Cardoso, "Web-based virtual reality with a-frame," in Proceedings of the 14th Iberian Conference on Information Systems and Technologies (CISTI), pp. 1-2, IEEE, Coimbra, Portugal, 2019.

[42] M. J. Prins, S. N. B. Gunkel, H. M. Stokking, and O. A. Niamut, "TogetherVR: a framework for photorealistic shared media experiences in 360-degree VR," SMPTE Motion Imaging Journal, vol. 127, no. 7, pp. 39-44, 2018.

[43] S. Neelakantam and T. Pant, "Introduction to a-frame," Learning Web-Based Virtual Reality, Apress, Berkeley, CA, USA, 2017.

[44] Cordova, 2021, https://cordova.apache.org/.

[45] K. L. vNorman, B. Shneiderman, B. Harper, and L. Slaughter, Questionnaire for User Interaction Satisfaction, University of Maryland, College Park, MD, USA, 1989. 